\title{
Effects of solvent, excitation wavelength, and concentration on the photobehavior of some diazonaphthoquinones
}

\author{
Marco Cipolloni, ${ }^{a}$ Pier L. Gentili, ${ }^{a}$ Fausto Ortica, ${ }^{a}$ Ralph S. Becker, \\ Gianna Favaro ${ }^{a^{*}}$ \\ ${ }^{a}$ Università di Perugia, Dipartimento di Chimica, 06123 Perugia, Italy \\ ${ }^{b}$ Locke Ave. Fort Worth, Texas 76116 USA \\ E-mail: favaro@unipg.it
}

\begin{abstract}
Diazonaphthoquinones (DNQs) are the photoactive components of resists used in the fabrication of integrated circuits. Exposed to radiation these compounds release molecular nitrogen undergoing a ring contraction (Wolff rearrangement). In this work the photobehavior of three compounds bearing two and three chromophoric DNQ units has been studied. Photochemical quantum efficiencies of reactant disappearance have been determined in acetonitrile at $290 \mathrm{~K}$ and in ethanol/methanol (4/1, $\mathrm{v} / \mathrm{v}$ ) solid matrix at $80 \mathrm{~K}$, using some irradiation wavelengths and different DNQ concentrations. The results obtained show that the course of the photoreaction strongly depends on the medium, the concentration, the temperature and the excitation wavelength.
\end{abstract}

Keywords: Diazonaphthoquinones, photochemistry, photokinetics, quantum yields

\section{Introduction}

One of the main purposes of our research has been to find new photochemical reactions having wavelength-dependent quantum yields. We have experimentally demonstrated that some photochromic compounds, especially those belonging to the class of chromenes, exhibit vibronic effects in their photochemistry. ${ }^{1,2}$ Also, bromo-derivatives of anthracene show a remarkable wavelength-dependent photo-behvior. ${ }^{3}$ These findings may offer a possibility to control photochemical and photophysical processes in fundamental as well as in applied research.

With the aim of discovering new photoreactions with wavelength-dependent photochemical yield, in this work we focused on the photo-induced detachment of nitrogen from diazonaphthoquinones (DNQs). Diazonaphthoquinones are compounds widely used in the fabrication of integrated circuits in microlithography ${ }^{4-7}$ where they are embedded in a novolak matrix (a phenol-formaldehyde condensation polymer). Exposed to radiation the diazoquinones release molecular nitrogen undergoing a ring contraction, the so-called Wolff rearrangement. ${ }^{8}$ The ketene produced, in the presence of water, yields a dienol which tautomerizes to carboxylic acid, scheme $1 .^{9}$ 


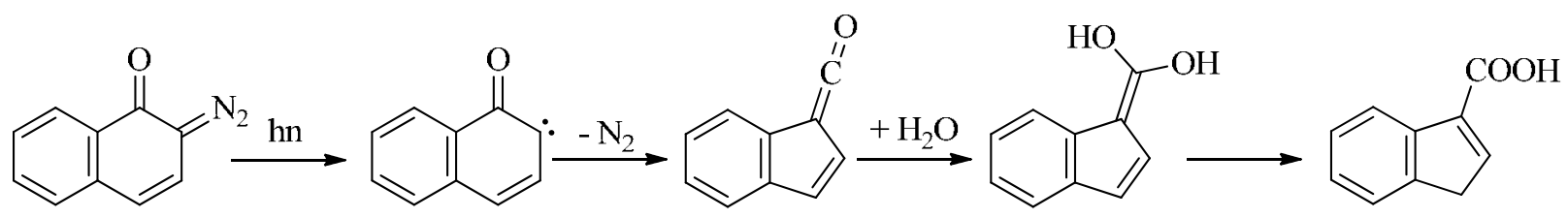

Scheme 1. The Wolff rearrangement.

The exposed regions of the photoresist film become soluble in the developer thus inducing the formation of a relief image while the unexposed region is insoluble. ${ }^{10-12}$

Numerous investigations have been carried out on this class of compounds, aimed at understanding the intimate mechanism of the photoreaction. Recent experiments point to an ultrafast, concerted mechanism, ${ }^{13}$ while calculations carried out by others suggest that the process may be asynchronous and the initial phase is dominated by nitrogen elimination. ${ }^{14}$

In this work, three DNQs were characterized from the spectroscopic and photochemical points of view. The effects of the solvent, excitation wavelength, and concentration on their photochemistry were investigated in order to discover whether their photoreactions could be modulated by changing some experimental factor. The compounds studied are (scheme 2): 2,4-bis(1,2-dihydro-2-diazo-1-oxonaphthalene-5-sulfonyloxy) benzophenone (BisDNQ); 1,3,5-tris-(1,2naphthoquinone-2-diazide-5-sulfonyloxy)-benzene (TrisDNQ-Sym) and 1,2,4-tris-(1,2naphthoquinone-2-diazide-5-sulfonyloxy)-benzene (TrisDNQ-uSym).

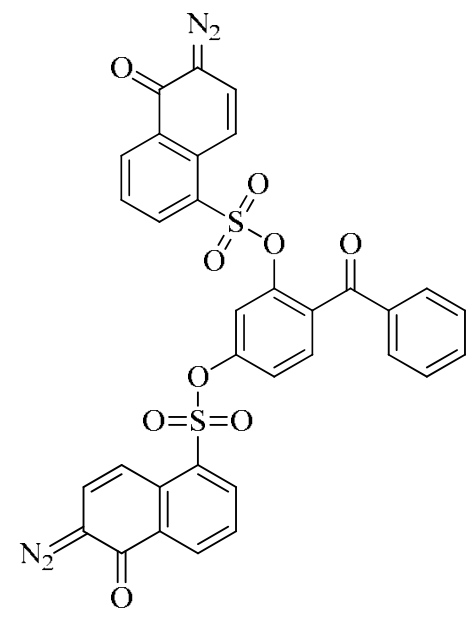

BisDNQ

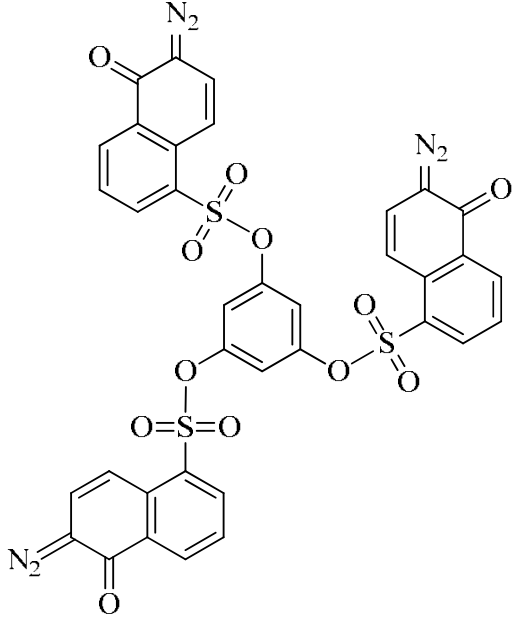

TrisDNQ-Sym<smiles>N=C1C=Cc2c(cccc2S(=O)(=O)Oc2ccc(OS(=O)(=O)c3cccc4c3C=CC(=N)C4=O)c(OS(=O)(=O)c3cccc4c3C=CC(=N)C4=O)c2)C1=O</smiles>

TrisDNQ-uSym

Scheme 2. Structures of the compounds investigated.

These molecules should allow us to investigate the effect on photochemistry of the number and position of the diazochromophores. The BisDNQ has been already studied in Novolak by photochemical methods. ${ }^{15}$ To the best of our knowledge, the TrisDNQs here studied were never 
photochemically investigated before, although other compounds bearing three DNQ units have been already considered. ${ }^{10,16,17}$ It is worthwhile noting that the trichromophoric resists are of particular interest in lithography since they are expected to give a higher image contrast and definition. ${ }^{10,18}$

\section{Results and Discussion}

\section{Absorption spectra}

The electronic absorption spectra of the three molecules studied extend from the UV to the blue visible region: their solutions appear pale yellow. No fluorescence emission was observed. The lowest energy absorption band which develops between 300 and $450 \mathrm{~nm}$ shows a broad structure that becomes slightly sharper in a rigid matrix at low temperature. Based on semiempirical calculations, performed using the MNDO procedure, several fairly intense electronic transitions were found to crowd in this spectral region, therefore a vibronic structure is not recognizable. The spectra of the three compounds in ethanol/methanol $(4 / 1, \mathrm{v} / \mathrm{v})$ at room temperature and the calculated electronic transitions are shown in Figure 1.

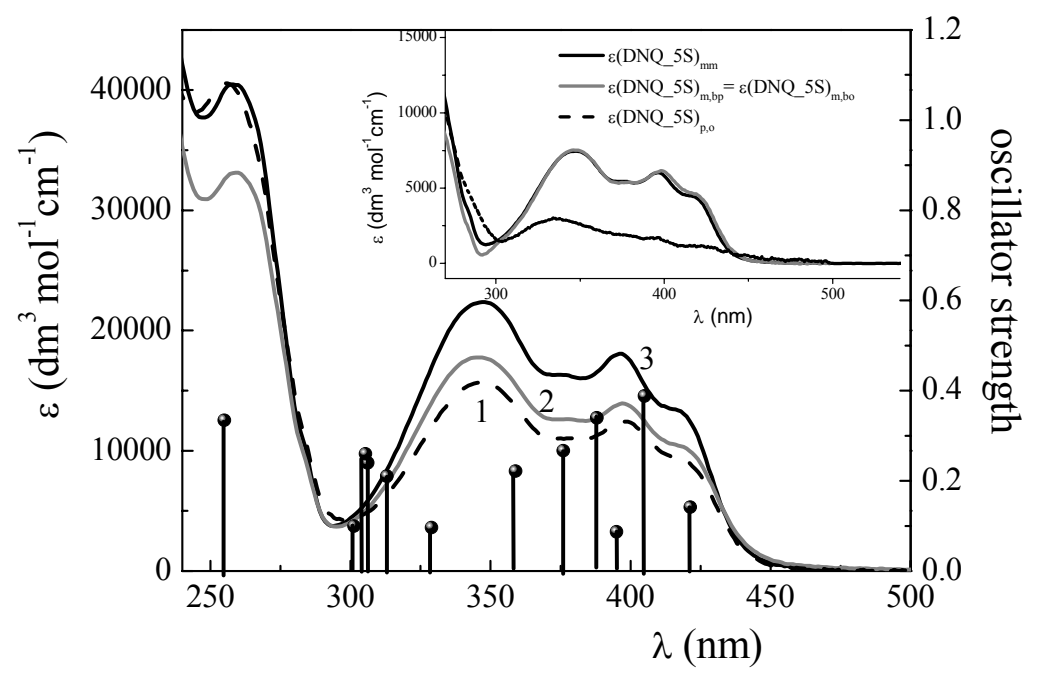

Figure 1. Absorption spectra of the three compounds in ethanol/methanol $(1 / 4, \mathrm{v} / \mathrm{v})$ at $\mathrm{room}$ temperature. 1: BisDNQ; 2: TrisDNQ-uSym; 3: TrisDNQ-Sym, and (•) semiempirical calculated spectrum of BisDNQ. Inset: contribution to the absorption spectra of the individual chromophores.

The orbitals involved in the lowest energy electronic transition having a consistent oscillator strength value $(\mathrm{HOMO} \rightarrow \mathrm{LUMO}+1)$ are represented in Figure 2 for a model molecule (methyl 6diazo-5,6-dihydro-5-oxo-naphthalene-1-sulfonate). It can be observed that the electronic rearrangement occurring on excitation is in favor of the release of nitrogen in the first reaction step. 


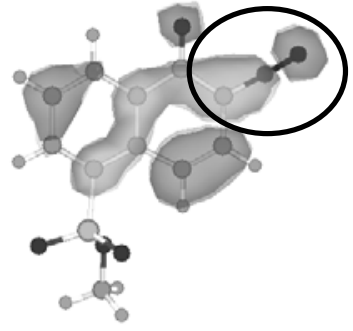

HOMO

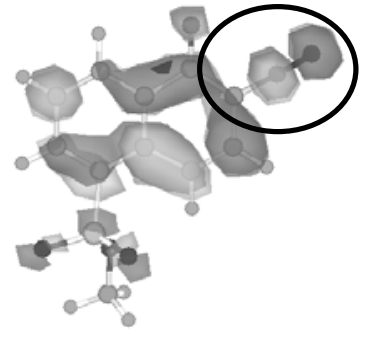

$\mathrm{LUMO}+1$

Figure 2. HOMO and LUMO+1 orbitals for the model molecule methyl 6-diazo-5,6-dihydro-5-oxonaphthalene-1-sulfonate; circles highlight the reactive site.

Molar absorption coefficients and wavelengths of the main peaks in different media are reported in Table 1 . The spectral shapes are scantly affected by the solvent and structure. The $\varepsilon$ values for the low energy band range from 10000 to $20000 \mathrm{dm}^{3} \mathrm{~mol}^{-1} \mathrm{~cm}^{-1}$, higher energy transitions peaked around $\sim 230$ and $\sim 260 \mathrm{~nm}$ are by far more intense $\left(\varepsilon \sim 40000 \mathrm{dm}^{3} \mathrm{~mol}^{-1} \mathrm{~cm}^{-1}\right)$. As expected the $\varepsilon$ values for BisDNQ are lower than for TrisDNQs. The absorption spectrum of BisDNQ is similar to that recorded in methanol. ${ }^{15}$ An appreciable, solvent-dependent substituent effect can be perceived in the $\varepsilon$ values. From a first glance at the spectra in Figure 1, TrisDNQ-Sym exhibits the largest molar absorption coefficients above $300 \mathrm{~nm}$ and BisDNQ the smallest. For the band centered at 259 $\mathrm{nm}$, the $\varepsilon$ values of BisDNQ are equivalent to those of TrisDNQ-Sym and larger than those of TrisDNQ-uSym.

Table 1. Molar absorption coefficients of the DNQs at the maximum wavelengths, $\varepsilon / \mathrm{dm}^{3} \mathrm{~mol}^{-1} \mathrm{~cm}^{-1}$ $(\lambda / \mathrm{nm})$, in selected solvents at room temperature $(290 \mathrm{~K})$ and $80 \mathrm{~K}$

\begin{tabular}{|c|c|c|c|c|}
\hline \multirow[t]{2}{*}{ compd } & \multirow{2}{*}{$\begin{array}{l}\mathrm{MeCN} \\
290 \mathrm{~K} \\
\end{array}$} & \multirow{2}{*}{$\frac{\mathrm{EtOH} / \mathrm{H}_{2} \mathrm{O}(1 / 2, \mathrm{v} / \mathrm{v})}{290 \mathrm{~K}}$} & \multicolumn{2}{|c|}{$\mathrm{MeOH}-\mathrm{EtOH}(1 / 4, \mathrm{v} / \mathrm{v})$} \\
\hline & & & $290 \mathrm{~K}$ & $80 \mathrm{~K}$ \\
\hline \multirow[t]{5}{*}{ BisDNQ } & $48000(226)$ & $54500(227)$ & $50000(227)$ & \\
\hline & $38600(256)$ & $45500(260)$ & $40500(257)$ & \\
\hline & $15800(352)$ & $17900(343)$ & $15500(345)$ & $14500(354)$ \\
\hline & $12100(396)$ & $13900(397)$ & $12450(399)$ & $11600(398)$ \\
\hline & $8400(420)$ & $10300(420)$ & $9000(420)$ & $8900(422)$ \\
\hline \multirow[t]{4}{*}{ TrisDNQ-uSym } & $33100(260)$ & & & \\
\hline & $21900(353)$ & & $19000(345)$ & $20000(355)$ \\
\hline & $16700(396)$ & & $14700(399)$ & $15000(399)$ \\
\hline & $11500(420)$ & & $10900(420)$ & $11000(421)$ \\
\hline \multirow[t]{4}{*}{ TrisDNQ-Sym } & $40400(260)$ & & & \\
\hline & $18600(353)$ & & $22300(345)$ & $22400(355)$ \\
\hline & $14500(396)$ & & $17700(399)$ & 17600 (399) \\
\hline & $9900(420)$ & & $12800(420)$ & $13100(421)$ \\
\hline
\end{tabular}


Because of the structural similarities of the three compounds, their spectral properties can be interpreted by partitioning the individual molecules into different chromophoric units. They can be conceived as tetrachromophoric compounds. TrisDNQ-Sym consists of three equivalent 1-oxo-2diazo-1,2-dihydronaphtalene-5-sulfonate (DNQ_5S) units, anchored to a benzene moiety, each having two DNQ units in meta positions. Following a simplified procedure, we can express the molar absorption coefficient of TrisDNQ-Sym as the sum of the $\varepsilon$ values of the three equivalent DNQ_5S moieties, $\varepsilon\left(\mathrm{DNQ} \_5 \mathrm{~S}\right)_{\mathrm{m}, \mathrm{m}}$, and that of benzene, i.e., Equation 1:

$$
\varepsilon(\text { Tris DNQ }-S y m)=3 \varepsilon\left(D N Q \_5 S\right)_{m, m}+\varepsilon(\text { benzene })
$$

The molar absorption coefficient of TrisDNQ-uSym can be expressed as the sum of four contributions, Equation 2:

$$
\varepsilon(\text { TrisDNQ }-u S y m)=\varepsilon\left(D N Q_{-} 5 S\right)_{m, p}+\varepsilon\left(D N Q_{-} 5 S\right)_{m, o}+\varepsilon\left(D N Q_{-} 5 S\right)_{p, o}+\varepsilon(\text { benzene })
$$

where $\varepsilon(\mathrm{DNQ} 5 \mathrm{SS})_{\mathrm{m}, \mathrm{p}}$ is the molar absorption coefficient of a DNQ unit bounded to a benzene having two other DNQ units in meta and para positions, respectively; $\varepsilon(\mathrm{DNQ} 5 \mathrm{~S})_{\mathrm{m}, \mathrm{o}}$ refers to a DNQ_5S unit having two other units in meta and ortho positions, respectively, and $\varepsilon\left(\mathrm{DNQ} \_5 \mathrm{~S}\right)_{\mathrm{p}, \mathrm{o}}$ is that of a DNQ_5S unit with two units in para and ortho, respectively.

Likewise, the molar absorption coefficient of BisDNQ can be expressed as the sum of contributions by four chromophores, Equation 3:

$$
\varepsilon(\text { BisDNQ })=\varepsilon\left(D N Q \_5 S\right)_{m, b p}+\varepsilon\left(D N Q_{-} 5 S\right)_{m, b o}+\varepsilon(\text { benzoyl })+\varepsilon(\text { benzene })
$$

In Equation 3, $\varepsilon\left(\mathrm{DNQ} \_5 \mathrm{~S}\right)_{\mathrm{m}, \mathrm{bp}}$ and $\varepsilon\left(\mathrm{DNQ} \_5 \mathrm{~S}\right)_{\mathrm{m}, \mathrm{bo}}$ are the absorption coefficients of two DNQ_5S units with a DNQ_5S unit in meta position and a benzoyl group in para and ortho position, respectively. The sum $\varepsilon$ (benzoyl) $+\varepsilon$ (benzene) is the molar absorption coefficient of benzophenone. Because of the similarity of electronic effects in ortho and para positions, it can be assumed that:

$$
\varepsilon\left(D N Q_{-} 5 S\right)_{m, p} \approx \varepsilon\left(D N Q_{-} 5 S\right)_{m, o}
$$

and $\quad \varepsilon\left(D N Q_{-} 5 S\right)_{m, b p} \approx \varepsilon\left(D N Q_{-} 5 S\right)_{m, b o}$

Furthermore, since benzoyl and DNQ moieties are conjugative substituents, it can be assumed that:

$$
\varepsilon\left(D N Q_{-} 5 S\right)_{m, p} \approx \varepsilon\left(D N Q_{-} 5 S\right)_{m, b p}
$$

Based on Equations 1, 2 and 3 and assumptions in 4, 5, and 6, and taking into account the contribution of benzophenone but ignoring that of benzene at $\lambda>240 \mathrm{~nm}$, the contributions to the 
overall $\varepsilon$ value by the individual chromophores, $\varepsilon\left(\mathrm{DNQ}_{-} 5 \mathrm{~S}\right)_{\mathrm{m}, \mathrm{m}}, \varepsilon\left(\mathrm{DNQ} \_5 \mathrm{~S}\right)_{\mathrm{m}, \mathrm{bp}}$ and $\varepsilon\left(\mathrm{DNQ} \_5 \mathrm{~S}\right)_{\mathrm{p}, \mathrm{o}}$, can be evaluated. The results are shown in the inset of Figure 1. In the spectral region between 300 and $450 \mathrm{~nm}, \varepsilon\left(\mathrm{DNQ}_{-} 5 \mathrm{~S}\right)_{\mathrm{m}, \mathrm{m}}$ and $\varepsilon\left(\mathrm{DNQ}_{-} 5 \mathrm{~S}\right)_{\mathrm{m}, \mathrm{bp}}$ are almost equivalent and significantly larger than $\varepsilon\left(\mathrm{DNQ} \_5 \mathrm{~S}\right)_{\mathrm{p}, \mathrm{o}}$. On the other hand, for the band centered at $259 \mathrm{~nm}$, the value of $\varepsilon(\mathrm{DNQ} 5 \mathrm{~S})_{\mathrm{p}, \mathrm{o}}$ is the highest whereas that of $\varepsilon\left(\mathrm{DNQ}_{-} 5 \mathrm{~S}\right)_{\mathrm{m}, \mathrm{bp}}$ is the lowest. In the latter region, the $\varepsilon$ of BisDNQ is high because of the strong contribution by the $\pi, \pi^{*}$ transition of benzophenone.

For some structurally analogous model molecules (o-diazobenzoquinone, o-diazonaphthoquinone, and o-diazonaphthoquinone-5-sulfonic acid) calculations at the MS-CASPT2 level have been performed by Blancafort et al. ${ }^{14}$ in order to assign the experimental UV-vis spectra. It has been calculated that the lowest energy $\pi, \pi^{*}$ state has a non-planar minimum characterized by an out-of-plane bending of the diazo group and a stretching of the C-N bond. These active modes are in agreement with the expectation that the initial step of the reaction is dominated by nitrogen elimination (see also Figure 2).

\section{Photochemistry}

The photoreaction time course of the compounds under study is significantly affected by the operational conditions, that is, the solvent, the excitation wavelength, the temperature, and the concentration. These effects were tested preliminarily in order to choose the best conditions under which quantitative determinations of photochemical yields for DNQ disappearance would be the most reliable.

Effects of the solvent and temperature. These compounds are scantly soluble in non-polar solvents, therefore they were studied in polar ones: $\mathrm{MeCN}$, an ethanol-water $(2 / 1, \mathrm{v} / \mathrm{v})$ mixture, and an ethanol/methanol $(4 / 1, \mathrm{v} / \mathrm{v})$ mixture. The latter medium was chosen in order to be able to perform measurements in a rigid matrix at $80 \mathrm{~K}$. The spectral behaviors upon irradiation were very similar for the three compounds, therefore, graphical examples reported below for one of them will represent the behavior of any of the others under the same conditions.

Figure 3 shows that the spectral changes undergone upon irradiation with $350 \mathrm{~nm}$ light of TrisDNQ-uSym solutions depend on the solvent and temperature. On the other hand, keeping constant all experimental factors except the excitation wavelength, qualitatively different end-ofirradiation spectra resulted.

At $290 \mathrm{~K}$ the photoreaction is almost complete in $\mathrm{MeCN}$, apart from a hardly detectable and broad absorption around $480 \mathrm{~nm}$ (Figure 3a), whereas in the ethanol/methanol (4/1, v/v) mixture (Figure $3 \mathrm{~b}$ ) a colored byproduct, characterized by a broad and red-shifted absorption band ( $\lambda_{\max } \sim$ $520 \mathrm{~nm}$ ), is formed. However, irradiation of a rigid ethanol/methanol matrix at $80 \mathrm{~K}$ (Figure 3c) results in a significantly different end-of-irradiation spectrum, characterized by an intense absorption maximum at $293 \mathrm{~nm}$, which is also present at $290 \mathrm{~K}$ in ethanol/methanol mixture and in $\mathrm{MeCN}$, but at a much lower intensity. 


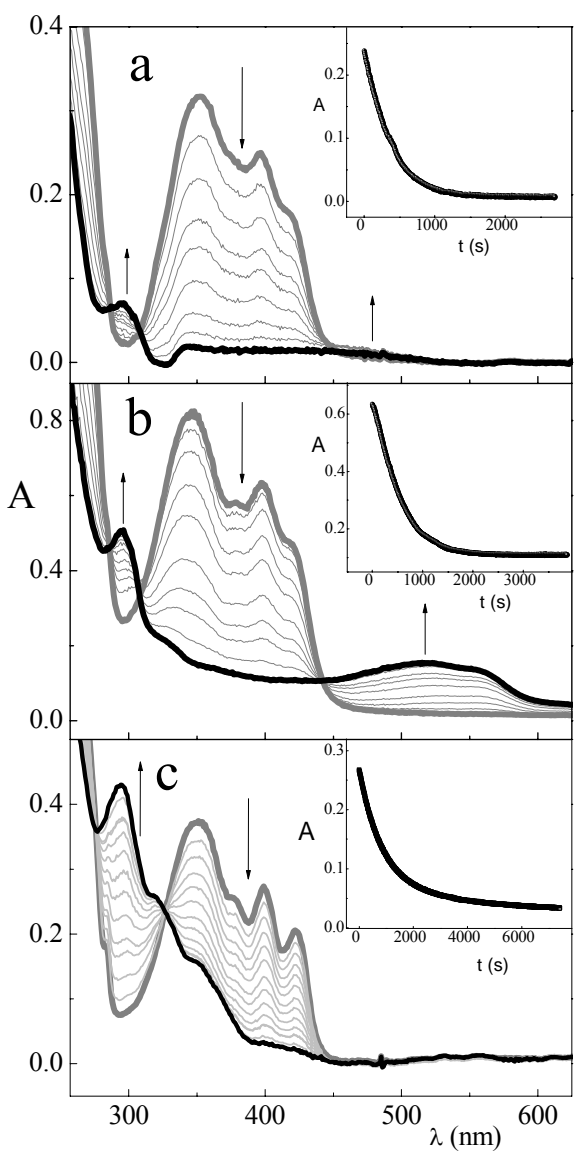

Figure 3. Spectral time evolution of the TrisDNQ-uSym upon steady state irradiation at $350 \mathrm{~nm}$ : gray and black solid lines indicate the spectrum before irradiation and at the end-of-irradiation, respectively. (a) $\mathrm{MeCN}$ solution at $290 \mathrm{~K}$; (b) ethanol/methanol (4/1, v/v) solution at $290 \mathrm{~K}$; (c) ethanol/methanol $(4 / 1, \mathrm{v} / \mathrm{v})$ solution at $80 \mathrm{~K}$ (solid matrix). Vertical arrows indicate the direction of absorbance evolution. Insets: kinetics of photoreaction.

In an ethanol-water solution $(2 / 1, \mathrm{v} / \mathrm{v})$ at $290 \mathrm{~K}$ (not graphically reported here) the photoreaction appears clean up to the end of irradiation and the maximum at $293 \mathrm{~nm}$ is no longer present.

The irradiated solutions $(80 \mathrm{~K})$ were not thermally stable: when gradually warmed up to equilibrate at room temperature, degradation occurred leading to marked spectral variations in the UV and in the visible regions, as illustrated in Figure 4 for the BisDNQ. In the spectrum of the solutions undergoing heating (spectrum 2 in Figure 4), several bands appear which do not belong to DNQ and seem to characterize different chemical species formed under different experimental conditions. These bands have maxima located at $398 \mathrm{~nm}$, double-peak at 324-333 nm, and at 299 $\mathrm{nm}$. The spectrum of the equilibrated solution (spectrum 3 in Figure 4) results from the superimposition of the end-of-irradiation spectrum at $290 \mathrm{~K}$ and the spectrum of the reactant before irradiation. 
The thermal spectral evolution depended on the excitation wavelength used to bleach the photoresist: more degradation product(s) were formed at higher excitation energy.

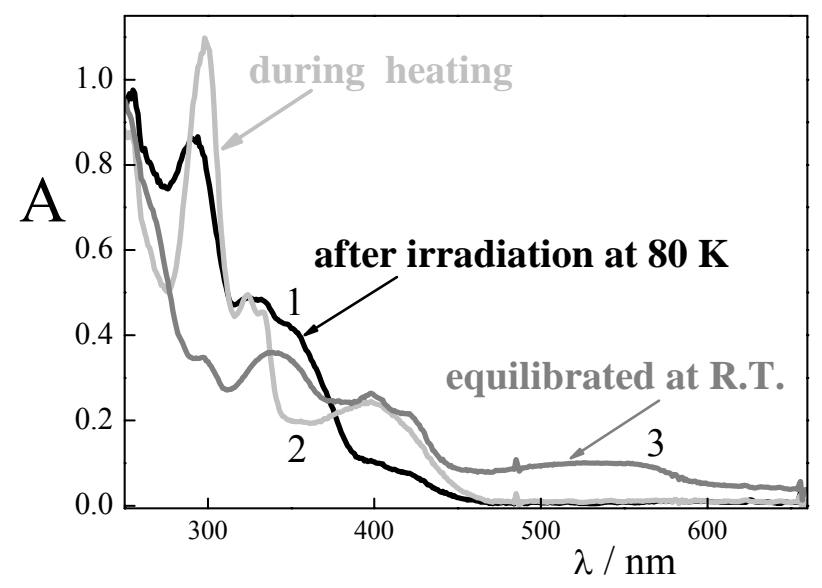

Figure 4. Absorption spectra of an ethanol/methanol (4/1, v/v) (1/4, v/v) BisDNQ solution at $80 \mathrm{~K}$, recorded after irradiation at $80 \mathrm{~K}$ (1, black), during heating (2, light gray), and after thermal equilibration to room temperature (3, gray).

Concentration effect. By increasing the concentration by one order of magnitude, from $2 \times 10^{-5}$ to $2 \times 10^{-4} \mathrm{~mol} \mathrm{dm}^{-3}$ in $\mathrm{MeCN}$, the spectrum at the end of the irradiation significantly changed. The weak color band $\left(\lambda_{\max } \sim 450 \mathrm{~nm}\right)$, observed upon irradiation of a MeCN dilute solution, notably increased in the more concentrated solution, as shown in Figure 5 where diluted and concentrated solutions are compared.

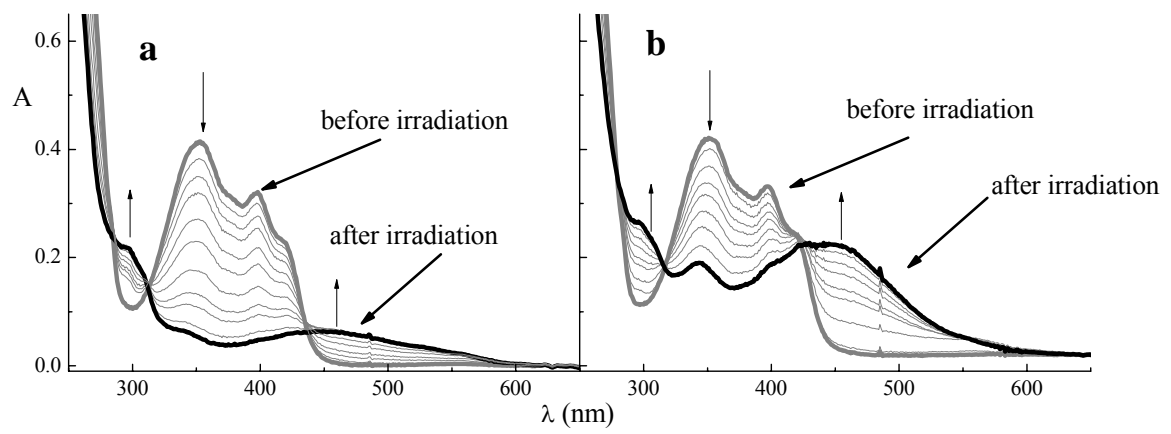

Figure 5. Spectral time evolution of BisDNQ upon irradiation with $399 \mathrm{~nm}$ light in $\mathrm{MeCN}$ at $290 \mathrm{~K}$ : gray solid line, before irradiation; black solid line, after irradiation. (a) $[\mathrm{BisDNQ}]=2 \times 10^{-5} \mathrm{~mol}$ $\mathrm{dm}^{-3}, 1 \mathrm{~cm}$ cell-path; (b) $\left[\right.$ BisDNQ] $=2 \times 10^{-4} \mathrm{~mol} \mathrm{dm}^{-3}, 0.1 \mathrm{~cm}$ cell-path. Vertical arrows highlight the direction of the spectral evolution. 
HPLC analysis of the irradiated concentrated solution revealed the presence of some unidentified chemical products emerging from the chromatographic column at different retention times (Figure 6) but having a similar electronic transition centered at $450 \mathrm{~nm}$ (see inset of Figure 6). These new products can be tentatively supposed to be oligomers of the DNQ molecules. The same chromophoric unit, which is present in all oligomers, is responsible for their absorption spectra whereas the oligomer size determines dipole moments/polarizability and therefore affects retention times.

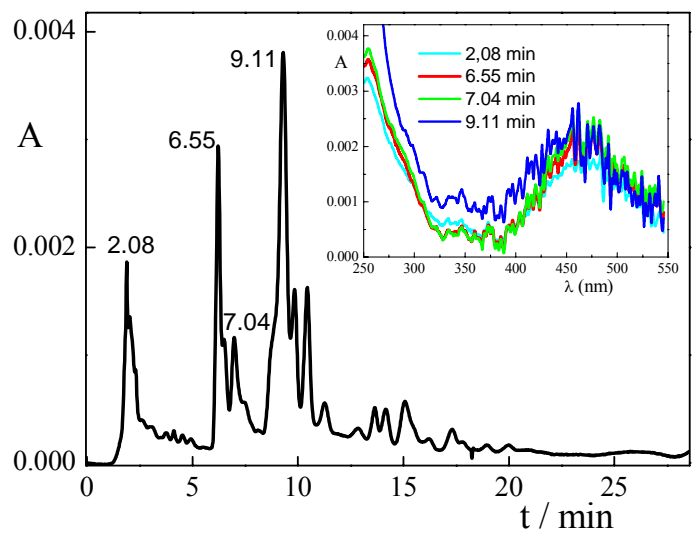

Figure 6. HPLC analysis of a $2 \times 10^{-4} \mathrm{~mol} \mathrm{dm}^{-3} \mathrm{MeCN}$ solution of BisDNQ after 4 hours irradiation with 399 nm light. Inset: normalized absorption spectra of four components.

Detection of photo-aggregation in the ground state in the $10^{-4}-10^{-5} \mathrm{~mol} \mathrm{dm}^{-3}$ concentration range hints the formation of some relatively long-lived (approximately estimated longer than $10 \mu \mathrm{s}$ ) intermediate species, which could be able to interact with the ground state DNQ, thus subtracting photosensitive material to the overall system.

Quantum yield determinations. The determinations of quantum yield $(\Phi)$ were performed using spectrophotometric and chromatographic methods. At $80 \mathrm{~K}, \Phi$ was only determined spectrophotometrically since, by heating the solutions up to room temperature, unwanted side reactions occurred (see Figure 4). This obviously made unsuited HPLC separations. Based on the above qualitative observations, we decided that the best conditions to obtain the most reliable quantum yield values of DNQ disappearance upon irradiation by the spectrophotometric method would be using relatively low concentrations $\left(\sim 2 \times 10^{-5} \mathrm{~mol} \mathrm{dm}^{-3}\right)$, to avoid aggregation, and performing the experimental runs in media where degradation was less evident, i.e., in $\mathrm{MeCN}$ solution. Experiments in an aqueous alcoholic solution $\left(\mathrm{EtOH} / \mathrm{H}_{2} \mathrm{O} 2: 1\right)$ were also carried out at room temperature, in order to obtain information about the role of water in the photoreaction. Excitation wavelengths were chosen based on the spectral features of the steady-state absorption spectra. The results are shown in Tables 2 and 3. All reported yields are averaged on at least three independent experimental determinations. In $\mathrm{MeCN}$, where two experimental methods 
(spectrophotometric and chromatographic) were used, the results from both were in agreement. This means that the quantum yield of DNQ disappearance is not concentration dependent.

Table 2. Quantum yields of DNQ conversion in MeCN (if not otherwise indicated) at $290 \mathrm{~K}$ (accuracy within 5\%)

\begin{tabular}{lllll}
\hline Compd & $\lambda_{\text {exc }}=254 \mathrm{~nm}$ & $\lambda_{\text {exc }}=350 \mathrm{~nm}$ & $\lambda_{\text {exc }}=399 \mathrm{~nm}$ & $\lambda_{\text {exc }}=423 \mathrm{~nm}$ \\
\hline TrisDNQ-Sym & 0.32 & 0.57 & 0.33 & 0.31 \\
TrisDNQ-uSym & 0.27 & 0.55 & 0.36 & 0.26 \\
BisDNQ & 0.36 & 0.32 & 0.34 & 0.36 \\
BisDNQ(EtOH $\left./ \mathrm{H}_{2} 02 / 1\right)$ & 0.22 & 0.22 & 0.31 & 0.39 \\
\hline
\end{tabular}

Table 3. Quantum yields of DNQ conversion in ethanol/methanol (4/1, v/v) at $80 \mathrm{~K}$ (accuracy within $10 \%$ )

\begin{tabular}{llll}
\hline Compd & $\lambda_{\text {exc }}=350 \mathrm{~nm}$ & $\lambda_{\text {exc }}=399 \mathrm{~nm}$ & $\lambda_{\text {exc }}=423 \mathrm{~nm}$ \\
\hline TrisDNQ-Sym & 0.24 & 0.30 & 0.20 \\
TrisDNQ-uSym & 0.24 & 0.15 & 0.15 \\
BisDNQ & 0.20 & 0.13 & 0.10 \\
\hline
\end{tabular}

From the foregoing it can be easily understood that the systems investigated and their photoreactions are very difficult to interpret and to control. This occurs because the first intermediates and/or photoproduct(s) can differently and promptly react with several surrounding species included the solvent and the starting reactant itself.

Literature studies on structurally similar DNQs suggest two different reaction paths: (i) a concerted reaction mechanism which has been proposed on the basis of nanosecond ${ }^{19,20}$ and picosecond $^{13,21}$ time-resolved spectroscopy, and (ii) an asynchronous process initiated by nitrogen elimination that has been suggested based on computational results ${ }^{14}$ but also proposed in experimental studies performed using nanosecond ${ }^{21}$ and ultrafast ${ }^{22}$ time resolved spectroscopy. It has been demonstrated ${ }^{22,23}$ that in some cases a branched mechanism is possible: the ketene is formed in both a fast process $(\mathrm{t}<0.4 \mathrm{ps})$ from the diazoketone excited state (concerted Wolff rearrangement) and a slower process $(700 \mathrm{ps})$ from the relaxed singlet ketocarbene. The ketene was detected for structurally similar molecules by Delaire et al. ${ }^{24}$ using UV-vis laser photolysis techniques and by Scaiano et al. using IR detection. ${ }^{25}$ The precursor(s) of ketene that could be a carbene $^{26,27}$ or an oxirene ${ }^{12,21}$ are not easily detectable experimentally because of their short lifetimes, mainly controlled by solute-solvent interactions. Both species can evolve to the ketene which is very reactive and participates in various (cyclo)additions, ${ }^{28}$ included the reaction with itself in competition with other reactive substrates to form diketene.

As regarding our results, by comparing the end-of-irradiation spectra in an ethanol/methanol matrix (Figure 3c, dotted lines) with the spectrum obtained by Varma et al. [see Figure 8c in ref. 27] for 4-tert-butylphenyl-2-diazo-1-oxo-1,2-dihydronaphthalene-5-sulfonate in an ethanol glass at 77 
$\mathrm{K}$ and assigned to the ketene, a close similarity is observed. Based on this comparison, in this work, the ketene was also recognized in fluid solution, where its presence was strongly influenced by the experimental conditions, such as the solvent and the intensity and duration of exposure to light. In the presence of substantial amounts of water $\left(\mathrm{EtOH} / \mathrm{H}_{2} \mathrm{O}, 2: 1\right)$ the ketene was completely undetectable, due to increased rate of the acid forming process by interaction of ketene with excess water. ${ }^{29}$ In acetonitrile, the reaction was slowed down due to the scant water amount, present in the commercial solvent as a trace, and the ketene was still recognizable from the maximum at $293 \mathrm{~nm}$.

The thermal evolution of the spectra observed on warming solutions irradiated in a matrix at 80 $\mathrm{K}$ (Figure 4, spectrum 2) evidences the characteristics of ketene. Upon thermal equilibration, colored product(s) appear(s) that can be mainly attributed to reaction(s) of the ketene with the solvent, leading to formation of esters. ${ }^{28}$

The band that we noted between 450 and $550 \mathrm{~nm}$, whose intensity increased with increasing DNQ concentration, was not revealed by others. Based on the concentration effect, we attribute this band to different compounds resulting from azocoupling reactions ${ }^{30,31}$ which require diffusion controlled encounters and therefore cannot take place in a rigid medium. The $293 \mathrm{~nm}$ absorption band that we attribute to the ketene is scarcely developed in Figure $5 \mathrm{~b}$ because of its interaction with the ground state DNQ.

The quantum yields of DNQ consumption in $\mathrm{MeCN}$ at room temperature are fairly high, especially for the TrisDNQs. There is a peak in quantum efficiency (greater than $50 \%$ ) with $350 \mathrm{~nm}$ excitation for the two TrisDNQs whereas the yield of the BisDNQ is somewhat lower and approximately constant over the whole excitation wavelength range. In contrast, in an ethanol/water mixture, the quantum yield for BisDNQ disappearance increases on going from short to longer wavelengths. The $\Phi$ values (0.22-0.38) determined by us are in good agreement with the number $(0.23)$ reported by Schuster ${ }^{15}$ for BisDNQ in novolak using $404 \mathrm{~nm}$ excitation wavelength. For other structurally similar compounds the quantum yields range from 0.3 to $0.54 .{ }^{27}$ In a rigid matrix (ethanol/methanol, 4/1, v/v) lower yields were determined, the difference between the Bis and TrisDNQs was maintained, but the excitation wavelength effect was no longer observed.

\section{Conclusions}

To the best of our knowledge, this study is the first one in which the photochemistry of the TrisDNQs has been investigated quantitatively in different media at various excitation wavelengths. The results obtained show that the solvent, the concentration and the temperature significantly affect the course of the photoprocess. The photoreaction is fairly efficient $(\Phi \sim 0.2 \div 0.6)$ but is difficult to control due to its extreme sensitivity to the surrounding ambient and temperature. When all other experimental conditions are kept constant, the quantum yield of the reaction depends on the excitation wavelength.

Intervention of thermal side processes, during or after irradiation, generate colored products that can negatively affect the performance in applications. However, it is noteworthy that the photochemistry can be performed throughout a wide UV-vis spectral range, making it possible 
using a variety of laser sources for excitation, and that its effectiveness can be modulated by changing the excitation wavelength.

\section{Experimental Section}

Materials. All of the molecules investigated (Scheme 2) required careful HPLC purifications. The BisDNQ and the TrisDNQs were kindly supplied by St Jean (Canada). The latter were in a mixture of 1,2,4-trihydroxybenzene (TrisDNQ-uSym) and 1,3,5-trihydroxybenzene (TrisDNQ-Sym) esterified with 2-diazo-1-naphthol-5-sulfonyl chloride. The two isomers were separated by HPLC and their structures were distinguished by ${ }^{1} \mathrm{H}$ NMR, from the different spectral features of the central benzene hydrogens, as illustrated in Figure 7. The solvents, acetonitrile (MeCN), ethanol $(\mathrm{EtOH})$ and methanol $(\mathrm{MeOH})$, Fluka products, were used as received. The trans-stilbene, used as a standard in HPLC determinations, was purchased from BDH.
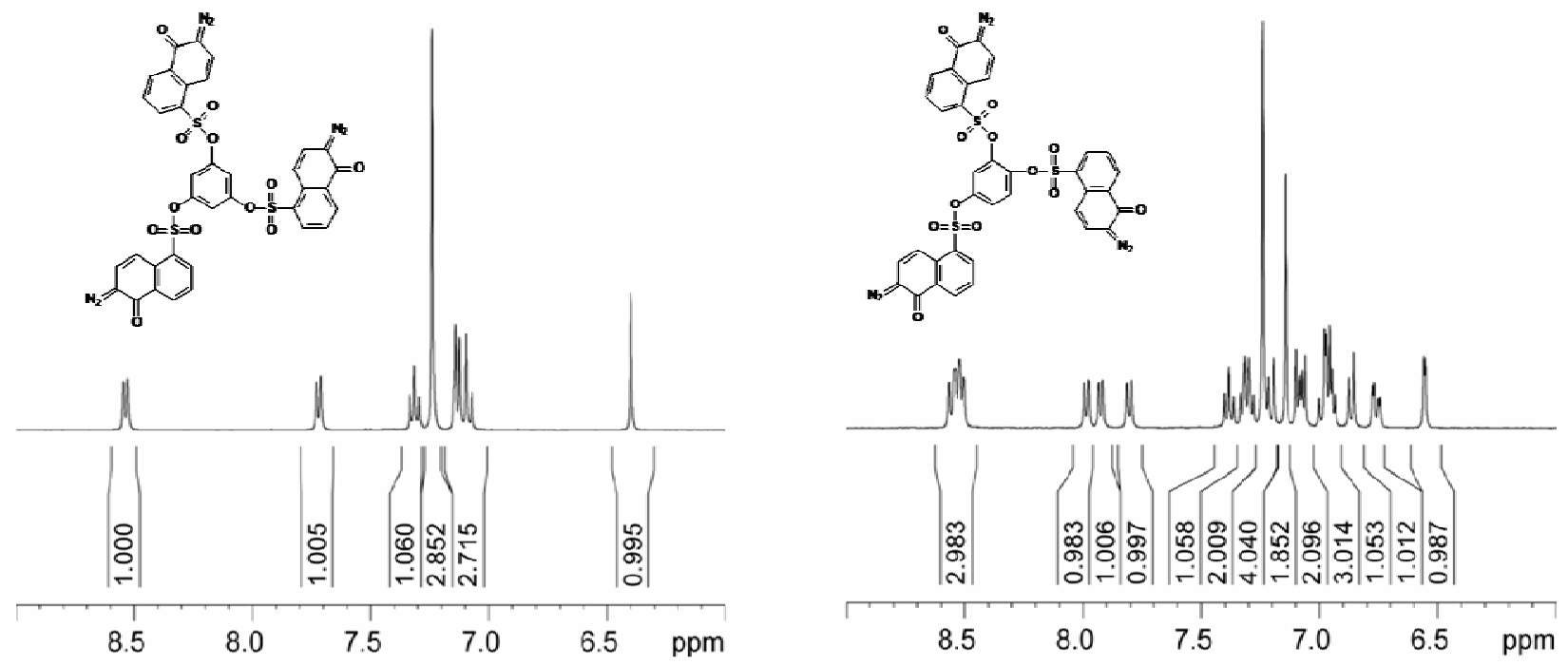

Figure 7. One-dimensional ${ }^{1} \mathrm{H}-\mathrm{NMR}$ spectra of TrisDNQ-Sym and TrisDNQ-uSym in $\mathrm{CDCl}_{3}$ at room temperature.

Equipment. The absorption spectra were recorded using a HP 8453 diode-array spectrophotometer or a Perkin Elmer $\lambda 800$ spectrophotometer. For purifications and separations of the compounds, a HPLC system, equipped with Waters 600 pump and controller, 2487 dual $\lambda$ absorbance and 996 photodiode array detectors, was used. A MeCN/water mixture (90/10 v/v) was used as eluent. An Altima C18 column was chosen as the stationary phase. For structural investigations, ${ }^{1} \mathrm{H}$ NMR spectra were recorded at room temperature by using a Bruker Avance DRX 400 spectrometer. Chemical shifts were referred to the residual resonance of the deuterated solvent, $\mathrm{CDCl}_{3}(\delta=7.27)$ and were reported in Figure 7 as ppm relative to tetramethylsilane. 
Reaction quantum yield. Irradiations of the sample solutions were carried out in a $1 \mathrm{~cm}$ (room temperature) and in a $0.85 \mathrm{~cm}(80 \mathrm{~K})$ cell-path. A $125 \mathrm{~W}$ Xe lamp coupled with a Jobin-Yvon H10UV monochromator, or a $500 \mathrm{~W} \mathrm{Hg}$ source coupled with an interference filter at $254 \mathrm{~nm}$, were used for irradiation. An Oxford Instruments cryostat was used for the temperature control. The determinations of quantum yields of DNQ disappearance were performed by two different methods. For the solid matrices, a spectrophotometric approach was applied, whereas for the liquid solutions both spectrophotometric and chromatographic methods were followed.

Spectrophotometric quantum yield determination. The radiation intensity (which was constant during each run) was determined using potassium ferrioxalate actinometry. It ranged from $2 \times 10^{-8}$ to $4 \times 10^{-7}$ Einstein $\mathrm{dm}^{-3} \mathrm{~s}^{-1}$ depending on the wavelengths. The concentrations of the starting solutions were in the order of $(1-4) \times 10^{-5} \mathrm{~mol} \mathrm{dm}^{-3}$ which correspond to absorbances in the range $0.1-0.6$ at the irradiation wavelengths. The monitoring beam was at right angle with respect to the irradiating beam, directed onto the cell using a fiber optic system. A gray filter (10\% transmittance) was inserted between the sample and the spectrophotometer source to avoid the possibility that a fraction of the probe light might contribute to the photodegradation of the sample. To determine the quantum yield, $\Phi$, of the process (7), i.e. the photo-induced disappearance of DNQ,

$$
\mathrm{DNQ} \stackrel{\mathrm{h} v}{\longrightarrow} \mathrm{P}
$$

an initial rate method was used, Equaton 8,

$$
\Delta \mathrm{A} / \Delta \mathrm{t}=\left(\varepsilon_{\mathrm{P}}-\varepsilon_{\mathrm{DNQ}}\right) \times \Phi \times \mathrm{I}^{0}\left(1-10^{-\mathrm{A}_{0}{ }^{\prime}}\right)
$$

where $\varepsilon_{\mathrm{DNQ}}$ and $\varepsilon_{\mathrm{P}}$ are the molar absorption coefficients of the reactant (DNQ) and the product (P), respectively, $\mathrm{I}^{0}$ is the intensity of the irradiating light and $\mathrm{A}_{0}{ }^{\prime}$ is the absorbance of the reactant at the irradiating wavelength. The determinations of $\Phi$ were carried out at low conversion percentage $(<$ $10 \%)$ wherein the decrease of absorbance of DNQ $(\Delta \mathrm{A})$ with time $(\Delta \mathrm{t})$ was linear. Under these conditions it can be assumed that the incident light absorbed by the photoproduct was negligible. From the plot of A vs t, $\Phi$ was determined. An example of such treatment is shown in Figure 8. 


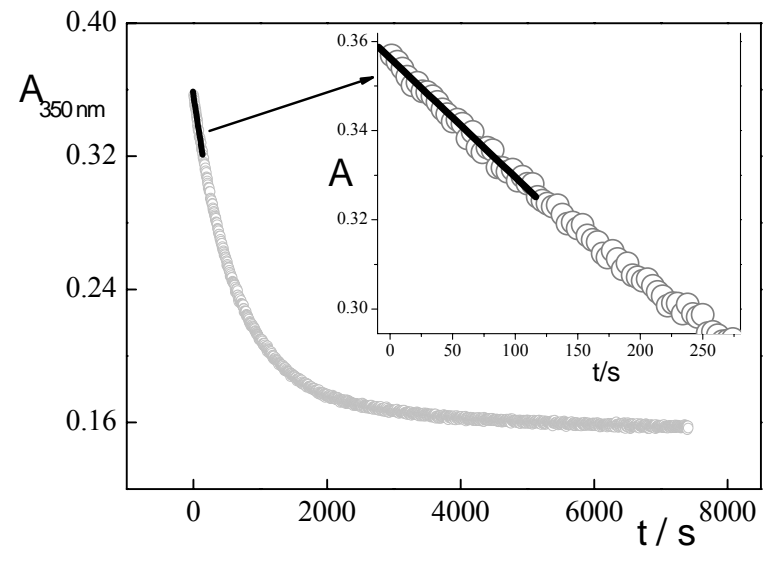

Figure 8. Example of photofading kinetics $\left(\lambda_{\text {anal }}=350 \mathrm{~nm}\right)$ for $1.8 \times 10^{-5} \mathrm{~mol} \mathrm{dm}^{-3}$ TrisDNQ-uSym in a ethanol/methanol $(4 / 1, \mathrm{v} / \mathrm{v})$ matrix at $80 \mathrm{~K}$. Black segments indicate the time-range considered for the quantum yield determination.

HPLC quantum yield determination. This type of measurements was carried out for all compounds at room temperature in acetonitrile and for BisDNQ also in ethanol/water 2/1 (v/v). Probe concentrations were of the order of $10^{-4} \mathrm{~mol} \mathrm{dm}^{-3}$ to assure total absorption of the irradiation light. Experimental data were collected using the HPLC system coupled to a 2487 dual $\lambda$ absorbance photomultiplier and a diode array recorder. Irradiation was carried out at 423, 399 and $350 \mathrm{~nm}$, using the Xenon source coupled to the monochromator, and at $254 \mathrm{~nm}$ using the $\mathrm{Hg}$ lamp coupled with an interferential filter. Irradiation times were such to promote conversion percentages in the $10 \%-20 \%$ range. Two samples from the same solution, with the addition of a suitable internal standard (trans-stilbene), were used to record chromatograms of the irradiated and non irradiated system. By comparing the areas of the chromatographic peaks normalized to the internal standard, the conversion percentage (\%) was evaluated, Equation 9

$$
\%=\left[\left(\mathrm{Ar} / \mathrm{Ar}_{\mathrm{st}}\right)-\left(\mathrm{Ar}^{\prime} / \mathrm{Ar}^{\prime}{ }_{\mathrm{st}}\right)\right] /\left(\mathrm{Ar} / \mathrm{Ar}_{\mathrm{st}}\right)
$$

where $\mathrm{Ar}$ and $\mathrm{Ar}_{\mathrm{st}}$ are the areas of the chromatographic peaks of the sample and the standard, respectively, in the non irradiated systems, whereas $\mathrm{Ar}^{\prime}$ and $\mathrm{Ar}_{\mathrm{st}}$ ' refer to the irradiated samples. The quantum yield $(\Phi)$ was obtained through Equation 10:

$$
\Phi=\% \times C_{0} V / I^{0} \Delta t
$$

where $C_{0}$ is the initial reactant concentration, $V$ is the irradiated volume, $I^{0}$ (Einstein $\mathrm{s}^{-1}$ ) is the actinometrically determined intensity of light that is completely absorbed by DNQ, and $\Delta t$ the irradiation time interval. Two channels of analysis were used: one that records the chromatogram at 
a fixed wavelength (generally $330 \mathrm{~nm}$ ) and the other that records the tri-dimensional chromatogram (absorbance $v s \lambda$ and time) over the entire wavelength range.

\section{Acknowledgements}

The authors thank for their valuable help L. Rocchigiani with NMR measurements and interpretation and D. Pannacci with HPLC analyses and separations. M. Cipolloni is grateful to the Regione Umbria for a fellowship.

\section{References}

1. Becker, R. S.; Pelliccioli, A. P.; Romani A.;. Favaro, G. J. Am. Chem. Soc. 1999, 121, 2104.

2. Gentili, P. L.; Romani, A.; Becker, R. S.; Favaro, G. Chem. Phys. 2005, 309, 167.

3. Favaro, G.; di Nunzio, M.R.; Gentili, P.L.; Romani, A.; Becker, R. S. J. Phys. Chem. A 2007, $111,5948$.

4. Kirmse, W. Eur. J. Org. Chem. 2002, 2193.

5. Reichmanis, E.; Novembre, A. E. Ann. Rev. Mater. Sci. 1993, 23, 11.

6. Reiser, A.; Shih, H.-Y.; Yeh, T.-F.; Huang, J.-P. Angew. Chem. Ed. Engl. 1996, 35, 2428.

7. Bowden, M. I.; Turner, S. R. Adv. Chem. Ser. 1988, $218,75$.

8. Wolff, L. Justus Liebigs Ann. Chem. 1902, 325, 129.

9. Andraos, J.; Kresge, A. J.; Popik, V. V. J. Am. Chem. Soc. 1994, 116, 961.

10. Reiser, A.; Huang, J.P.; He, X.; Yeh, T.F.; Jha, S.; Shih, H.Y.; Kim, M.S.; Han, Y.K.; Yan, K. Eur. Polymer J. 2002, 38, 619.

11. Shih, H-Y.; Reiser, A. Macromolecules 1996, 29, 2082.

12. Tanigaki, K.; Ebbesen, T. W. J. Phys. Chem. 1989, 93, 4531.

13. Wolpert, D.; Schade, M.; Brixner, T. J. Chem. Phys. 2008, 129, 094504.

14. Quansong, L.; Migani, A.; Blancafort, L. J. Phys. Chem. A 2009, 113, 9413.

15. Schuster, C.; Bendig, J.; Luger, P.; Weber, M.; Koch, A. J. Photochem. Photobiol. A: Chem. 1993, 69, 313.

16. Sauer, E.; Bendig, J.; Kertscher, U. Proc. Indian Acad. Sci. (Chem. Sci.) 1995, 10, 889.

17. Trefonas, P.; Daniels, P. K. Proc. SPIE 1987, 771, 194.

18. Han, Y. K.; Yan, Z.; Reiser, A. Macromolecules 1999, 32, 8421.

19. Barra, M.; Fisher, T. A.; Cernigliaro, G. J.; Sinta, R.; Scaiano, J. C. J. Am. Chem. Soc. 1992, $114,2630$.

20. Andraos, J.; Chiang, Y.; Huang, C.-G.; Kresge, A. J.; Scaiano, J. C. J. Am. Chem. Soc. 1993, 115, 10605.

21. Rosenfeld, A.; Mitzner, R.; Baumbach, B.; Bendig, J. J. Photochem. Photobiol. A: Chem. 1990 55, 259.

22. Burdzinski, G. T.; Wang, J.; Gustafson, T. L.; Platz, M. S. J. Am. Chem. Soc. 2008, 130, 3746.

23. Burdzinski, G.; Platz, M. S. J. Phys. Org. Chem. 2010, 23, 308. 
24. Delaire, J. A.; Faure, J.; Hassine-Renou, F.; Soreau, M. Nouv. J. Chim. 1987, 11, 15.

25. Camara de Lucas, N.; Netto-Ferreira, J. C.; Andraos, J.; Lusztyk, J.; Wagner, B. D.; Scaiano, J. C. Tetrahedron Lett. 1997, 38, 5147.

26. Meier, H.; Zeller, K. P. Angew. Chem. Inter. Ed. Engl. 1975, 14, 32.

27. Vleggaar, J. J. M.; Huizer, A. H.; Kraakman, P. A.; Nijssen, W. P. M.; Visser, R. J.; Varma, C. A. G. O. J. Am. Chem. Soc. 1994, 116, 11754.

28. Tidwell, T. T. Eur. J. Org. Chem. 2006, 563.

29. Andraos, J.; Kresge, A. J. J. Photochem. Photobiol. A: Chem. 1991, 57, 165.

30. Schuster, C.; Bendig, J. Prakt. Chem. 1991, 333, 91.

31. Protsenko, N. P. Zh. Org. Khim. 1977, 13, 1466. 